\section{Uso de suplementos dietéticos entre residentes do Município de São Paulo, Brasil}

\author{
Use of dietary supplements among inhabitants of \\ the city of São Paulo, Brazil
}

\author{
El uso de suplementos dietéticos en habitantes \\ de São Paulo, Brasil
}

1 Faculdade de Saúde
Pública, Universidade de
São Paulo, São Paulo, Brasil.
2 Instituto de Medicina
Social, Universidade do
Estado do Rio de Janeiro,
Rio de Janeiro, Brasil.
Correspondência
K. H. Brunacio
Faculdade de Saúde Pública,
Universidade de São Paulo.
Rua Vicente Rodrigues Sales
107, Taubaté, SP 12091-846,
Brasil.
kahh_honorato@yahoo.com.br

\section{Abstract}

This cross-sectional population-based study in the city of São Paulo, Brazil, aimed to assess prevalence of use of dietary supplements according to life stage and socio-demographic and behavioral characteristics. Prevalence of supplement use was low (6.35\%) among the 865 individuals interviewed, with a higher prevalence among women (PR =1.88; 95\%CI: 1.08-1.25). However, no statistically significant difference was found for the other variables. Combined vitamin and mineral supplements headed the list. Intake of supplements is still low in this population. However, the current weak evidence of benefits from regular use and the risks of excessive consumption justify monitoring the use of supplements.

Population Groups; Nutrition Surveys; Dietary Supplements
Karoline Honorato Brunacio 1

Eliseu Verly-Jr 2

Chester Luiz Galvão Cesar 1

Regina Mara Fisberg 1

Dirce Maria Marchioni 1

\section{Resumo}

Trata-se de estudo transversal de base populacional com residentes do Município de São Paulo, Brasil, que objetivou avaliar a prevalência do uso de suplementos dietéticos, segundo estágio de vida e características sociodemográficas e comportamentais. Observou-se baixa prevalência do uso de suplementos (6,35\%) entre os 865 indivíduos entrevistados, havendo maior prevalência entre mulheres $(R P=1.88$; IC95\%: 1,08-1,25). Não foi encontrada diferença estaticamente significante para as demais variáveis. Suplementos compostos por vitamina(s) e mineral(is) combinados lideraram o uso; no entanto, o consumo de suplementos pode ainda ser considerado baixo na população estudada. Tendo em vista as fracas evidências atuais de benefícios obtidos pelo uso regular de suplementos, em conjunto com os riscos para o consumo excessivo, justifica-se o monitoramento e vigilância do uso de suplementos na população.

Grupos Populacionais; Inquéritos Nutricionais; Suplementos Dietéticos 


\section{Introdução}

A dieta deve ser a forma prioritária para a obtenção dos nutrientes, mas o uso de suplementos dietéticos sob a forma medicamentosa tem-se tornado popular, sendo elevado nos Estados Unidos 1 e em países europeus 2. Evidências sobre os efeitos desse consumo são controversas $3,4,5,6$, porém é reconhecido que a ingestão excessiva de alguns nutrientes pode acarretar agravos à saúde. Há informações de níveis de ingestão seguros somente para alguns nutriente 3 , portanto a ingestão de quantidades além daquela ofertada por uma dieta equilibrada é questionável.

A relevância da informação sobre o consumo de suplementos dietéticos está relacionada não somente aos níveis seguros de ingestão de nutrientes, como também à sua inclusão em estudos dietéticos, para aferir a ingestão total de nutrientes.

O objetivo do estudo foi investigar a prevalência do uso de suplementos por parte dos moradores da cidade de São Paulo, tendo em vista a escassez desses dados no Brasil.

\section{Métodos}

Participantes do Inquérito de Saúde no Município de São Paulo em 2003 (ISA-Capital 2003) foram contatados entre 2007 e 2008. Foram localizados e concordaram em participar de inquérito alimentar 865 indivíduos (232 adolescentes, 343 adultos e 291 idosos). Detalhes da metodologia estão no Boletim ISA-Capital 2008 (http://www.fsp.usp. br/isa-sp/index_arquivos/Page3157.htm). Para comparar esta amostra com a inicial, verificou-se se a perda era diferencial em relação aos setores, o que poderia demonstrar que setores em bairros mais abastados deixariam de ser representados. Observou-se que foram contemplados 60 setores censitários dos 61 utilizados na amostra inicial. Também não foram observadas diferenças entre as amostras de 2003 e 2007 quanto à escolaridade do chefe da família, selecionada por representar nível socioeconômico.

A coleta de dados foi feita em entrevista domiciliar. O peso foi aferido por balança eletrônica, tipo plataforma, capacidade para $150 \mathrm{~kg}$ e sensibilidade de $100 \mathrm{~g}$ (Tanita Corporation of America Inc., Arlington Heights, Estados Unidos). Para altura, utilizou-se estadiômetro com escala em milímetros (Seca Bodymeter, modelo 208, Hamburgo, Alemanha), seguindo-se procedimentos padronizados 7 . O índice de massa corporal (IMC) foi calculado para classificar o estado nutricional, segundo as propostas da Organiza- ção Mundial da Saúde (OMS) para adolescentes, adultos e idosos 7 .

As variáveis utilizadas foram: gênero, faixa etária, em anos (adolescentes: 12-19 anos; adultos: 20-59 anos; idosos: 60 ou mais), escolaridade do chefe da família (categorizada em Ensino Fundamental completo ou Ensino Médio incompleto ou mais), satisfação corporal ("satisfeito" ou "não satisfeito", segundo a resposta à questão: "Gostaria que seu peso fosse diferente do atual?") e nível de atividade física. O tempo de atividade física foi coletado pela versão longa do Questionário Internacional de Atividade Física (IPAQ) validado para a população brasileira ${ }^{8}$. Os indivíduos com atividade física de lazer semanal maior que 150 minutos foram classificados como ativos, e aqueles que não atingiram este valor, como insuficientemente ativos 9 .

Os participantes foram questionados se tomavam algo para suplementar sua alimentação (como vitaminas, minerais ou outros produtos), podendo responder: "não"; "sim, regularmente" ou "sim, mas não regularmente". Se a resposta fosse positiva, perguntava-se o nome, composição, dose, frequência e tempo de uso do suplemento. As informações nutricionais dos suplementos foram obtidas nos sites dos fabricantes ou pelos rótulos. A classificação foi feita com base na legislação sanitária 10,11,12,13.

Foram calculadas as prevalências de uso e diferenças entre as variáveis verificadas pelo teste qui-quadrado. Utilizou-se um modelo de regressão de Poisson para verificar fatores associados com a utilização de suplementos. $\mathrm{Na}$ análise múltipla, aquelas que apresentaram valor de $\mathrm{p}<0,20$ na análise bivariada foram incluídas no modelo como covariáveis.

\section{Resultados}

A Tabela 1 apresenta as características da população. A prevalência de uso de suplementos foi de $6,35 \%$, e, dentre as variáveis analisadas, diferença significativa foi observada apenas para gênero (Tabela 2).

$\mathrm{Na}$ análise múltipla, somente o gênero ajustado pela faixa etária permaneceu significante no modelo. Maior prevalência de uso foi observada no gênero feminino ( $R P=1,88$; IC95\%: $1,08-1,25)$.

A frequência diária de consumo foi relatada por $82,9 \%$ dos consumidores, seguida da frequência de "uma a seis vezes por semana" (12,2\%) e "menor que uma vez por semana" $(4,9 \%)$.

Dos 81 suplementos utilizados, vinte (25\%) não puderam ser classificados, pela insuficiência de informação do respondente para carac- 
Tabela 1

Distribuição de adolescentes, adultos e idosos, segundo gênero, estado nutricional, satisfação corporal, escolaridade do chefe da família e prática de atividade física. São Paulo, Brasil, 2008

\begin{tabular}{|c|c|c|c|c|c|c|}
\hline & \multicolumn{2}{|c|}{ Adolescentes } & \multicolumn{2}{|c|}{ Adultos } & \multicolumn{2}{|c|}{ Idosos } \\
\hline & $\mathbf{n}$ & $\%$ & $\mathrm{n}$ & $\%$ & $\mathbf{n}$ & $\%$ \\
\hline \multicolumn{7}{|l|}{ Gênero } \\
\hline Masculino & 116 & 50,00 & 165 & 48,10 & 133 & 45,70 \\
\hline Feminino & 116 & 50,00 & 178 & 51,90 & 158 & 54,30 \\
\hline \multicolumn{7}{|l|}{ Estado nutricional } \\
\hline Baixo peso e eutrofia & 161 & 82,99 & 193 & 61,86 & 139 & 57,44 \\
\hline Sobrepeso e obesidade & 33 & 17,01 & 119 & 38,14 & 103 & 42,56 \\
\hline \multicolumn{7}{|l|}{ Satisfação corporal } \\
\hline Satisfeito & 111 & 48,05 & 123 & 36,18 & 147 & 51,22 \\
\hline Não satisfeito & 120 & 51,95 & 217 & 63,82 & 140 & 48,78 \\
\hline \multicolumn{7}{|l|}{ Escolaridade do chefe da família } \\
\hline Ensino Fundamental completo & 108 & 48,21 & 165 & 49,40 & 232 & 80,28 \\
\hline Ensino Médio incompleto ou mais & 116 & 51,79 & 169 & 50,60 & 57 & 19,72 \\
\hline \multicolumn{7}{|l|}{ Atividade física } \\
\hline Insuficientemente ativo & 218 & 93,97 & 339 & 98,83 & 290 & 99,66 \\
\hline Ativo & 14 & 6,03 & 4 & 1,17 & 1 & 0,34 \\
\hline Todos & 232 & 26,79 & 343 & 39,61 & 291 & 30,60 \\
\hline
\end{tabular}

Tabela 2

Prevalência de uso de suplementos, segundo gênero, estado nutricional, faixa etária, satisfação corporal, escolaridade do chefe da família e prática de atividade física. São Paulo, Brasil, 2008

\begin{tabular}{|c|c|c|c|}
\hline Características & $\%(n)$ & IC95\% & Valor de $p$ * \\
\hline \multicolumn{4}{|l|}{ Gênero } \\
\hline Masculino & $4,35(18)$ & $2,38-6,32$ & 0,021 \\
\hline Feminino & $8,19(37)$ & $5,65-10,72$ & \\
\hline \multicolumn{4}{|l|}{ Estado nutricional } \\
\hline Baixo peso e eutrofia & $6,49(32)$ & $4,31-8,67$ & 0,621 \\
\hline Sobrepeso e obesidade & $7,45(19)$ & $4,22-10,69$ & \\
\hline \multicolumn{4}{|l|}{ Faixa etária } \\
\hline Adolescentes & $4,31(10)$ & $1,69-6,93$ & \\
\hline Adultos & $5,54(19)$ & $3,11-7,97$ & 0,072 \\
\hline Idosos & $8,93(26)$ & $5,65-12,22$ & \\
\hline \multicolumn{4}{|l|}{ Satisfação corporal } \\
\hline Satisfeito & $7,09(27)$ & $4,50-9,67$ & 0,475 \\
\hline Não satisfeito & $5,87(28)$ & $3,76-7,98$ & \\
\hline \multicolumn{4}{|l|}{ Escolaridade do chefe da família } \\
\hline Ensino Fundamental completo & $5,74(29)$ & $3,71-7,78$ & 0,361 \\
\hline Ensino Médio incompleto ou mais & $7,31(25)$ & $4,54-10,08$ & \\
\hline \multicolumn{4}{|l|}{ Atividade física } \\
\hline Insuficientemente ativo & $6,14(52)$ & $4,52-7,76$ & 0,088 \\
\hline Ativo & $15,79(3)$ & $0,00-32,66$ & \\
\hline Todos & $6,35(55)$ & $4,72-7,98$ & \\
\hline
\end{tabular}

IC95\%: intervalo de $95 \%$ de confiança.

* Referente ao teste de qui-quadrado. 
terizar o produto. No restante, foi observada a seguinte distribuição: 16\% "vitaminas isoladas ou associadas entre si"; $5 \%$ "minerais isolados ou associados entre si”; $20 \%$ "associações de vitamina(s) com mineral(is)"; $4 \%$ "suplemento energético para atletas"; $5 \%$ "suplemento protéico para atletas"; $5 \%$ "alimentos compensadores"; $5 \%$ "aminoácidos de cadeia ramificada"; $4 \%$ "alimentos com fins específicos para atletas"; $2 \%$ "alimentos para redução de peso por substituição total das refeições"; $4 \%$ "alimentos enriquecidos/fortificados ou alimentos simplesmente adicionados de nutrientes" (como alimentos vitaminados/nutritivos que conferem sabor); 5\% outros compostos.

\section{Discussão}

A prevalência de consumo de suplementos dietéticos na cidade de São Paulo foi baixa (6,35\%), com maior prevalência entre mulheres. Os valores encontrados foram similares aos observados na Grécia, Espanha e Itália 2, porém menores que nos Estados Unidos 1,7, Reino Unido, Suécia e Holanda 2. Suplementos compostos por vitaminas e minerais e multivitamínicos lideraram o uso, em concordância com a literatura prévia 1,14,15.

A maior prevalência de uso de suplementos entre mulheres é consistente com os achados de outros estudos 1,2,14,15. Uma possível explicação é a maior preocupação com a saúde no gênero feminino, visto que procuram atendimento médico e são usuárias de medicamentos com mais frequência que os homens 16 . Há ainda as reco- mendações específicas para mulheres, como suplementação com ácido fólico em idade reprodutiva e ferro durante a gestação. A prevalência de uso não diferiu das demais variáveis, em desacordo com o encontrado em outros estudos, que apontaram diferenças entre suplementação e atividade física $14,15,17,18$, faixa etária, escolaridade e estado nutricional 14,15.

Considerando que os suplementos dietéticos provavelmente não são utilizados apenas por pessoas que tenham necessidade aumentada, há o risco de a quantidade de nutrientes ingerida ultrapassar o limite de consumo máximo tolerável (UL). Em virtude de possíveis efeitos adversos à saúde, caracteriza-se, então, uma questão importante para a vigilância epidemiológica na população. Nos Estados Unidos, a ingestão de nutrientes por meio de suplementos acima da UL já foi observada 1 .

O forte apelo publicitário e a expectativa de resultados rápidos, que exijam pouco esforço para obtenção de metas, sejam estéticas, sejam de saúde, podem contribuir para o uso indiscriminado dessas substâncias por pessoas que desconhecem seus riscos. São necessários mais estudos, que avaliem conjuntamente a alimentação e os suplementos para o monitoramento da ingestão segura de nutrientes.

Por fim, uma implicação importante desses resultados é que, em face da baixa prevalência de uso, a informação sobre o uso de suplementos parece ser dispensável, pelo menos até o momento, na estimativa do consumo de nutrientes obtidos por inquéritos populacionais.

\section{Resumen}

Estudio transversal de base poblacional que abarcó a habitantes de São Paulo, Brasil, que tuvo por objetivo evaluar la prevalencia del consumo de suplementos dietéticos, según etapas de la vida y variables sociodemográficas y de comportamiento. Se encontró una baja prevalencia del uso de suplementos (6,35\%) entre las 865 personas entrevistadas, con una mayor prevalencia observada en las mujeres ( $R P=1,88$; IC95\%: 1,08-1,25). Sin embargo, no se observó ninguna diferencia estadísticamente significativa para las demás variables. Los suplementos con vitaminas y minerales fueron los más frecuentes. El uso de suplementos puede todavía ser considerado bajo en esta población. No obstante, dada la falta de evidencias sobre los beneficios obtenidos por el consumo regular de suplementos, junto a los riesgos de un consumo excesivo, se justificó el seguimiento y la vigilancia sobre el uso de suplementos en la población.

Grupos de Población; Encuestas Nutricionales; Suplementos Dietéticos 


\section{Colaboradores}

K. H. Brunacio e E. Verly-Jr contribuíram na concepção e desenho do estudo, análise e interpretação dos dados, redação do artigo e revisão final após a contribuição dos coautores. C. L. G. Cesar e R. M. Fisberg contribuíram na revisão crítica do conteúdo intelectual e aprovação final da versão para publicação. D. M. Marchioni contribuiu na concepção e desenho, análise e interpretação dos dados, redação e revisão crítica do conteúdo intelectual e aprovação final da versão para publicação.

\section{Agradecimentos}

Ao CNPq, pelo apoio financeiro (processo no 503128/ 2010-4), e à FAPESP, pela bolsa de iniciação científica concedida a K. H. Brunacio (processo o 2010/08598-4) e pelo apoio financeiro (processos no 2009/11239-9 2009/15831-0).

\section{Referências}

1. Bailey RL, Fulgoni VL 3rd, Keast DR, Dwyer JT. Examination of vitamin intakes among us adults by dietary supplement use. J Acad Nutr Diet 2012; 112:657-63.e4.

2. Skeie G, Braaten T, Hjartåker A, Lentjes M, Amiano P, Jakszyn P, et al. Use of dietary supplements in the European Prospective Investigation into Cancer and Nutrition calibration study. Eur J Clin Nutr 2009; 63 Suppl 4:S226-38.

3. Huang HY, Caballero B, Chang S, Alberg AJ, Semba $\mathrm{RD}$, Schneyer CR, et al. The efficacy and safety of multivitamin and mineral supplement use to prevent cancer and chronic disease in adults: a systematic review for a National Institutes of Health state-of-the-science conference. Ann Intern Med 2006; 145:372-85.

4. Mayne ST, Ferrucci LM, Cartmel B. Lessons learned from randomized clinical trials of micronutrient supplementation for cancer prevention. Ann Rev Nutr 2012; 32:369-90.

5. Martínez ME, Jacobs ET, Baron JA, Marshall JR, Byers T. Dietary supplements and cancer prevention: balancing potential benefits against proven harms. J Natl Cancer Inst 2012; 104:732-9.
6. Fairfield KM. ACP Journal Club. Daily multivitamin supplements did not reduce risk for major CV events over $>10$ years in men. Ann Intern Med 2013; 158:JC2-8.

7. World Health Organization. Physical status: the use e interpretation of anthropometry. Geneva: World Health Organization; 1995.

8. Matsudo S, Araujo T, Matsudo V, Andrade D, Andrade E, Oliveira LC, et al. Questionário internacional de atividade física (IPAQ): estudo de validade e reprodutibilidade no Brasil. Rev Bras Ativ Fís Saúde 2001; 6:5-18.

9. World Health Organization. Global strategy on diet, physical activity and health. Fifty-seventh World Health Assembly. Geneva: World Health Organization; 2004

10. Ministério da Saúde. Portaria no 32 de 13 de janeiro de 1998. Aprova o regulamento técnico para suplementos vitamínicos e ou de minerais. Diário Oficial da União 1998; 15 jan.

11. Agência Nacional de Vigilância Sanitária. Resolução RDC no 18, de 27 de abril de 2010. Dispõe sobre alimentos para atletas. Diário Oficial de União 2010; 28 abr. 
12. Agência Nacional de Vigilância Sanitária. Portaria no 30 , de 13 de janeiro de 1998. Aprova o regulamento técnico referente a alimentos para controle de peso. Diário Oficial da União 1998; 16 jan.

13. Agência Nacional de Vigilância Sanitária. Portaria no 31 , de 13 de janeiro de 1998. Aprova o regulamento técnico referente a alimentos adicionados de nutrientes essenciais. Diário Oficial da União 1998; 16 jan.

14. Foote JA, Murphy SP, Wilkens LR, Hankin JH, Henderson BE, Kolonel LN. Factors associated with dietary supplement use among healthy adults of five ethnicities: the multiethnic cohort study. Am J Epidemiol 2003; 157:888-97.

15. Rock CL. Multivitamin-multimineral supplements: who uses them? Am J Clin Nutr 2007; 85 Suppl:277S-9S
16. Travassos C, Viacava F, Pinheiro R, Brito A. Utilização dos serviços de saúde no Brasil: gênero, características familiares e condição social. Pan Am J Public Health 2002; 11:365-73.

17. Santos KMO, Barros Filho AA. Consumo de produtos vitamínicos entre universitários de São Paulo, SP. Rev Saúde Pública 2002; 36:250-3.

18. Pereira RF, Lajolo FM, Hirschbruch MD. Consumo de suplementos por alunos de academias de ginástica em São Paulo. Rev Nutr 2003; 16:265-72.

Recebido em 07/Ago/2012

Versão final reapresentada em 20/Mar/2013

Aprovado em 01/Abr/2013 